\title{
Correlación entre la densidad de perfusión y el grosor de la capa de células ganglionares en sujetos sin diabetes y en pacientes diabéticos sin retinopatía
}

\section{Correlation between perfusion density and ganglion cell layer thickness in non-diabetic subjects and diabetic patients without retinopathy}

\author{
Guillermo Serrato-Martín ${ }^{1}$, Dulce M. Razo-Blanco-Hernández ${ }^{2 *}$, Selma A. Somilleda-Ventura ${ }^{3} y$ \\ Virgilio Lima-Gómez ${ }^{1}$
}

${ }^{1}$ Servicio de Oftalmología, Hospital Juárez de México; ²División de Investigación, Hospital Juárez de México; ${ }^{3}$ Centro de Investigación Biomédica, Fundación Hospital Nuestra Señora de la Luz I.A.P. Ciudad de México, México

\begin{abstract}
Resumen
Objetivo: Comparar la correlación entre la densidad de perfusión y el grosor de la capa de células ganglionares en sujetos sin diabetes y diabéticos sin retinopatía. Método: Estudio observacional, retrospectivo, comparativo y transversal, en sujetos sin diabetes (grupo 1) y diabéticos sin retinopatía (grupo 2) evaluados mediante tomografía de coherencia óptica y angiotomografía de coherencia óptica (Cirrus con Angioplex). Se compararon entre grupos la densidad de perfusión por cuadrantes (mapa de $3 \times$ $3 \mathrm{~mm}$ ), el grosor de la capa de células ganglionares por sectores ( $U$ de Mann-Whitney) y la correlación entre variables por grupo (Rho de Spearman). Resultados: Se incluyeron 34 sujetos en el grupo 1 y 38 en el grupo 2. Las densidades de perfusión interna, completa, superior y nasal fueron menores en el grupo $2(p<0.05)$; el grosor de la capa de células ganglionares no difirió entre grupos en ningún sector. Hubo correlaciones débiles entre la densidad de perfusión interna y completa y los grosores de la capa de células ganglionares en el grupo 1; en el grupo 2 hubo correlaciones débiles entre la perfusión del cuadrante inferior y el grosor de los sectores inferior e inferior temporal, donde la densidad de perfusión no difirió de la del grupo 1. Conclusiones: La correlación entre la densidad de perfusión y el grosor de la capa de células ganglionares es débil en los sujetos sin diabetes y se pierde en los pacientes diabéticos sin retinopatía, porque en ellos solo la densidad de perfusión es menor.
\end{abstract}

Palabras clave: Células ganglionares. Correlación. Densidad de perfusión. Diabetes. Grosor de la capa de células ganglionares. Retina.

\section{Abstract}

Purpose: To compare the correlation between perfusion density and ganglion cell layer thickness in non-diabetic subjects and diabetics without retinopathy. Methods: Non-experimental, retrospective, comparative cross-sectional study in subjects without diabetes (group 1) and diabetics without retinopathy (group 2) evaluated with optical coherence tomography and optical coherence tomography angiography (Cirrus with Angioplex). We compared perfusion density between groups by quadrants $(3 \times 3 \mathrm{~mm}$

\section{Correspondencia:}

Dulce M. Razo-Blanco-Hernández

Avda. Instituto Politécnico Nacional, 5160

Col. Magdalena de las Salinas, G.A. Madero Fecha de recepción: 18-09-2020

C.P. 07760 , Ciudad de México, México

Fecha de recepción: 18-09-2020

Disponible en internet: 05-05-2021

E-mail: razoblanco.dulce@gmail.com

DOI: 10.24875/RMO.M21000153

Rev Mex Oftalmol. 2021;95(3):95-100

www.rmo.com.mx

0187-4519/๑ 2020 Sociedad Mexicana de Oftalmología. Publicado por Permanyer. Este es un artículo open access bajo la licencia CC BY-NC-ND (http://creativecommons.org/licenses/by-nc-nd/4.0/). 
map), ganglion cell layer thickness by sectors (Mann Whitney's $U$ ) and the correlation between variables within groups (Spearman's Rho). Results: Thirty-four subjects in group 1, 38 in group 2. Inner, total, superior and nasal perfusion densities were lower in group 2 ( $p<0.05)$; ganglion cell layer thickness did not differ between groups in any sector. There were weak correlations between inner and total perfusion densities and ganglion cell layer thicknesses in group 1; in group 2 there were weak correlations between inferior quadrant perfusion and ganglion cell layer thickness in the inferior and inferior temporal sectors, where perfusion density did not differ from group 1. Conclusions: The correlation between perfusion density and ganglion cell layer thickness is weak in non-diabetic subjects; diabetics without retinopathy show no correlation, observing only a lower perfusion density.

Key words: Ganglion cells. Correlation. Perfusion density. Diabetes. Ganglion cell layer thickness. Retina.

\section{Introducción}

La retinopatía diabética es una de las principales causas de ceguera prevenibles en sujetos en edad productiva ${ }^{1,2}$. Tradicionalmente se ha considerado como una enfermedad microvascular, específica de la diabetes; sin embargo, antes de que aparezcan sus manifestaciones clínicas, mediante tomografía de coherencia óptica de dominio espectral se han encontrado cambios en el grosor de la capa de células ganglionares ${ }^{3}$. Otra característica previa al desarrollo de la retinopatía diabética es la disminución en la densidad capilar parafoveal, identificada mediante angiografía por tomografía de coherencia óptica ${ }^{4,5}$; ambas características pueden coexistir. $\mathrm{Kim}$, et al. ${ }^{6}$ han reportado una correlación fuerte entre la progresión de los cambios del grosor de la capa de células ganglionares y la densidad vascular $(r=0.616-0.871)$, así como entre el grosor de la capa de células ganglionares y la densidad de perfusión $(r=0.371-0.555)^{6}$.

La reducción de la densidad capilar podría disminuir el grosor de la capa de células ganglionares, mas no se ha determinado la proporción en que contribuye a sus cambios antes de que aparezca la retinopatía diabética. Entre las variables angiotomográficas con el equipo Cirrus con Angioplex, la densidad vascular mide la longitud de los capilares que detecta, mientras que la densidad de perfusión mide el porcentaje del área de la mácula en la cual se detecta flujo capilar? La correlación entre la densidad de perfusión y el grosor de la capa de células ganglionares en distintas regiones de la mácula podría estimar la contribución de la primera a los cambios de la segunda.

El objetivo de este estudio fue comparar la correlación entre la densidad de perfusión y el grosor de la capa de células ganglionares en sujetos sin diabetes y en pacientes diabéticos sin retinopatía.

\section{Método}

Se realizó un estudio observacional, retrospectivo, comparativo y transversal en sujetos sin diabetes y pacientes con diabetes sin retinopatía diabética de la Ciudad de México y su área metropolitana. La muestra se obtuvo de pacientes atendidos en un hospital federal de referencia entre el 27 de enero y el 31 de enero del año 2020, durante una campaña de detección de enfermedades maculares mediante tomografía de coherencia óptica. El estudio se apegó a los principios de la Declaración de Helsinki y recibió autorización de las comisiones de investigación y ética en investigación, en la institución donde se realizó (registro HJM 0782/20-R).

Se incluyeron sujetos con una edad entre 20 y 80 años, de ambos sexos, sin diabetes o con diabetes sin retinopatía diabética, que contaran con un estudio de tomografía de coherencia óptica espectral (cubo de grosor macular) y uno de angiografía por tomografía de coherencia óptica (mapa de perfusión de $3 \times 3 \mathrm{~mm}$ ) de adecuada calidad, con intensidad de señal $>7$. Se eliminaron los casos cuyos estudios tuvieran una calidad de la imagen inadecuada o presentaran errores de medición, y aquellos en que la fotografía del fondo de ojo o la tomografía de coherencia óptica revelaran datos de cualquier retinopatía u otras enfermedades oculares.

Todos los participantes fueron evaluados bajo un protocolo estandarizado por un solo investigador, como se describe a continuación: medición de la agudeza visual corregida en equivalentes de Snellen, fotografías de fondo de ojo de $45^{\circ}$ bajo midriasis farmacológica (Visu Cam lite, Zeiss, Dublin, CA, USA) y medición del grosor del punto central, el volumen macular, el grosor de la capa de células ganglionares, la densidad de perfusión y las características de la zona avascular foveal, en un mapa de $3 \times 3 \mathrm{~mm}$, con el equipo HD-OCT Cirrus 5000 con Angioplex (Zeiss, Dublin, CA, USA), utilizando el algoritmo OMAG.

Los sujetos sin diabetes se asignaron al grupo $1 \mathrm{y}$ los pacientes con diabetes se asignaron al grupo 2. Se seleccionó un ojo por paciente, mediante un programa de números aleatorios.

Las variables en estudio fueron el grosor de la capa de células ganglionares, que se definió por la medición automática del espesor de la retina neurosensorial 
Tabla 1. Comparación de las variables basales entre grupos (mediana y rango intercuartílico)

\begin{tabular}{|c|c|c|c|}
\hline Variable & Grupo $1(n=34)$ & Grupo 2 ( $n=38)$ & p \\
\hline Edad (años) & $46(33.7-60.5)$ & $56(49.7-60.5)$ & $0.005^{*}$ \\
\hline Grosor del punto central $(\mu \mathrm{m})$ & $250(237.5-268)$ & $250.5(232-265.5)$ & $0.513^{*}$ \\
\hline Volumen macular $\left(\mathrm{mm}^{3}\right)$ & $10.1(9.9-10.5)$ & $9.9(9.7-10.3)$ & $0.222^{*}$ \\
\hline Área de la zona avascular foveal $\left(\mathrm{mm}^{2}\right)$ & $0.28(0.21-0.36)$ & $0.30(0.19-0.38)$ & $0.939^{*}$ \\
\hline Perímetro de la zona avascular foveal (mm) & $2.28(2.02-2.76)$ & $2.52(2.06-2.82)$ & $0.720^{*}$ \\
\hline Circularidad de la zona avascular foveal (\%) & $0.68(0.59-0.73)$ & $0.63(0.49-0.70)$ & $0.172^{*}$ \\
\hline Sexo femenino & $18(52.9 \%)$ & $24(63.2 \%)$ & $0.380^{\dagger}$ \\
\hline Hipertensión arterial & 0 & $10(26.3 \%)$ & $0.001^{\dagger}$ \\
\hline
\end{tabular}

delimitado por la capa plexiforme interna y la capa de fibras nerviosas, y la densidad de perfusión macular, la cual se definió como el porcentaje de la superficie de la mácula donde se identificaban capilares. La densidad de perfusión se midió por cuadrantes: superior, temporal, inferior y nasal. El grosor de la capa de células ganglionares se midió por sectores: superior, temporal superior, temporal inferior, inferior, nasal inferior y nasal superior. Se emplearon las mediciones de ambas variables que el equipo generó automáticamente.

Se realizó una prueba de Kolmogórov-Smirnov, que reveló una distribución anormal en algunas variables, por lo que los resultados se expresaron como medianas y rangos intercuartílicos. Se compararon las medianas del grosor de la capa de células ganglionares y de la densidad de perfusión entre los grupos mediante la prueba $U$ de Mann-Whitney; además, se determinó la correlación entre el grosor de la capa de células ganglionares en cada sector y la densidad de perfusión en cada cuadrante, en cada grupo, mediante la prueba Rho de Spearman. Se consideró como significativo un valor de $p<0.05$. Los resultados se almacenaron y analizaron en el programa IBM SPSS Ver. 26 (SPSS Inc, Chicago, IL, USA).

\section{Resultados}

Se estudiaron 72 ojos de 72 sujetos, con una edad de 20 a 77 años (mediana: 54.5; rango intercuartílico: $43-60)$, y 42 eran del sexo femenino (58.3\%). El tiempo de evolución de la diabetes fue de 1 mes a 18 años (mediana: 5; rango intercuartílico: 2-12). Diez participantes padecían hipertensión arterial (13.9\%). Se asignaron 34 sujetos al grupo 1 y 38 al grupo 2. La comparación entre variables basales se presenta en la tabla 1; la mediana de edad y la frecuencia de hipertensión arterial fueron mayores en el grupo 2.

La tabla 2 muestra la comparación entre grupos de la densidad de perfusión; las medianas fueron menores en el grupo 2, a excepción de en los cuadrantes temporal e inferior, en los cuales no hubo diferencias entre los grupos. La densidad central mostró una tendencia a ser menor en el grupo 2. El grosor de la capa de células ganglionares no presentó diferencias entre grupos (Tabla 3).

Las correlaciones entre la densidad de perfusión y el grosor de la capa de células ganglionares en el grupo 1 se presentan en la tabla 4; hubo una correlación débil entre las densidades de perfusión interna y completa y los grosores de los sectores, a excepción del nasal superior. Los grosores del sector inferior presentaron una correlación débil con la densidad de perfusión del cuadrante inferior.

En el grupo 2 solo hubo una correlación débil entre las densidades de perfusión interna y completa, y el grosor de los sectores inferior y temporal inferior, que se correspondió con los cuadrantes donde la densidad de perfusión no difirió entre los grupos (Tabla 5).

\section{Discusión}

Se encontró una menor densidad de perfusión en los pacientes con diabetes sin retinopatía en comparación con los sujetos sanos; la diferencia fue mayor en los cuadrantes superior y nasal, lo cual fue suficiente para reducir tanto la densidad de perfusión interna como la 
Tabla 2. Comparación de la densidad de perfusión entre grupos (U de Mann-Whitney)

\begin{tabular}{|l|c|c|c|}
\hline Densidad de perfusión (\%) & Grupo $1(\mathrm{n}=34)$ & Grupo $2(\mathrm{n}=38)$ & 0.050 \\
\hline Central & $18.7(13.9-22.5)$ & $14.8(11.8-19.9)$ & 0.012 \\
\hline Interna & $40.5(37.0-41.6)$ & $37.8(35.9-39.3)$ & 0.018 \\
\hline Completa & $37.7(34.4-39.2)$ & $35.4(33.1-37.0)$ & 0.023 \\
\hline Superior & $41.0(36.4-41.8)$ & $37.7(33.7-39.5)$ & 0.55 \\
\hline Temporal & $40.3(37.2-41.4)$ & $39.2(36.7-41.3)$ & 0.09 \\
\hline Inferior & $39.1(37.2-41.8)$ & $38.4(35.3-39.6)$ & $37.6(35.1-39.7)$ \\
\hline Nasal & $39.7(37.5-42.6)$ & & 0.01 \\
\hline
\end{tabular}

Tabla 3. Comparación del grosor de la capa de células ganglionares entre grupos (U de Mann-Whitney)

\begin{tabular}{|l|c|c|c|}
\hline Grosor de la capa de células ganglionares $(\mu \mathrm{m})$ & Grupo $1(\mathrm{n}=34)$ & Grupo $2(\mathrm{n}=38)$ & 0.91 \\
\hline Superior & $83.0(77.7-86.2)$ & $82.5(77.0-88)$ & 0.29 \\
\hline Temporal superior & $81.5(77.0-86.0)$ & $80.0(77.2-84.0)$ & 0.59 \\
\hline Temporal inferior & $81.0(76.7-86.0)$ & $81.0(74.0-85.0)$ & 0.93 \\
\hline Inferior & $79.0(73.7-83.0)$ & $79.0(75.0-82.0)$ & 0.74 \\
\hline Nasal inferior & $81.0(75.7-86.0)$ & $79.5(75.7-86.2)$ & 0.67 \\
\hline Nasal superior & $83.5(78.8-88.5)$ & $83.0(77.7-88.2)$ & 0.5 \\
\hline
\end{tabular}

Tabla 4. Correlación entre la densidad de perfusión por cuadrante y el grosor de la capa de células ganglionares por sector en el grupo 1 ( $n=34$, Rho de Spearman)

\begin{tabular}{|l|c|c|c|c|c|c|}
\hline \multirow{2}{*}{ Densidad de perfusión } & \multicolumn{5}{|c|}{ Grosor de la capa de células ganglionares } \\
\cline { 2 - 6 } & Superior & Temporal superior & Temporal inferior & Inferior & Nasal inferior \\
\hline Interna & $0.388(0.023)^{*}$ & $0.357(0.038)^{*}$ & $0.403(0.018)^{*}$ & $0.416(0.014)^{*}$ & $0.426(0.012)^{*}$ \\
\hline Completa & $0.381(0.026)^{*}$ & $0.403(0.018)^{*}$ & $0.425(0.012)^{*}$ & $0.385(0.024)^{*}$ & $0.369(0.032)^{*}$ \\
\hline Inferior & NS & NS & $0.358(0.037)^{*}$ & $0.365(0.034)^{*}$ & $0.368(0.032)^{*}$ \\
\hline Nasal & NS & NS & NS & NS & $0.394(0.021)^{*}$ \\
\hline
\end{tabular}

*Valor de p.

NS: no significativo.

completa. El grosor de la capa de células ganglionares fue semejante en ambos grupos; las correlaciones entre la densidad de perfusión y el grosor de la capa de células ganglionares no pudieron compararse, porque la mayoría no fueron significativas en el grupo 2 .

Los valores del grosor de la capa de células ganglionares por sector en los sujetos sin diabetes del presente estudio fueron semejantes a los reportados por Lee, et al. ${ }^{8}$ en un estudio coreano, medidos con el mismo instrumento; los valores en sujetos con diabetes sin retinopatía fueron mayores que los reportados por ellos. Esta diferencia pudiera deberse a que su estudio solo incluyó pacientes con más de 10 años de evolución de la diabetes, lo cual podría reducir el grosor de la capa de células ganglionares; otra explicación podría ser una variabilidad poblacional, ya que se han reportado diferencias interraciales del grosor de la capa de células ganglionares ${ }^{9}$. 
Tabla 5. Correlación entre la densidad de perfusión por cuadrante y el grosor de la capa de células ganglionares por sector en el grupo 2 ( $n=38$, Rho de Spearman)

\begin{tabular}{|l|c|c|c|c|c|}
\hline \multirow{2}{*}{ Densidad de perfusión } & \multicolumn{5}{|c|}{ Grosor de la capa de células ganglionares } \\
\cline { 2 - 7 } & Superior & Temporal superior & Temporal inferior & Inferior & Nasal inferior \\
\hline Interna & NS & NS & $0.388(0.016)^{*}$ & $0.336(0.039)^{*}$ & NS \\
\hline Completa & NS & NS & $0.384(0.017)^{*}$ & $0.326(0.046)^{*}$ & NS \\
\hline Temporal & NS & NS & $0.403(0.012)^{*}$ & NS & NS \\
\hline
\end{tabular}

*Valor de p.

NS: no significativo.

En los sujetos sin diabetes se encontró una correlación débil entre la densidad de perfusión interna y el grosor de la capa de células ganglionares en la mayoría de los sectores. Esto indica que la variación de la densidad de perfusión no explica en una proporción considerable los cambios del grosor de la capa de células ganglionares. La mayoría de estas correlaciones débiles no se observaron en el grupo 2, a excepción de en los sectores donde la densidad de perfusión no mostró diferencias entre los grupos.

Kim, et al. ${ }^{6}$ reportaron una correlación fuerte entre la disminución del grosor de la capa de células ganglionares y cuatro parámetros de angiografía por tomografía de coherencia óptica en sujetos con diabetes, sin retinopatía o con retinopatía diabética no proliferativa. Su estudio longitudinal mostró cambios progresivos en un grupo que tenía ya reducción tanto del grosor de la capa de células ganglionares como de la densidad de perfusión, medidos con el mismo equipo que empleamos en nuestro estudio. En el presente estudio solo se incluyeron pacientes sin retinopatía diabética con un corto tiempo de evolución de la diabetes, lo cual explicaría que no se hallaran cambios en el grosor de la capa de células ganglionares, aun cuando la densidad de perfusión hubiera disminuido. Tampoco se encontraron las correlaciones que el mismo grupo ${ }^{10}$ reportó entre el grosor de la capa de células ganglionares y la zona avascular foveal, porque en este estudio las medidas de esa región fueron semejantes entre los grupos.

En este estudio transversal, la menor densidad de perfusión en los pacientes con diabetes se presentó sin que existieran cambios en el grosor de la capa de células ganglionares. Para esta etapa de progresión del daño por diabetes, la densidad de perfusión no tuvo correlación con el grosor de la capa de células ganglionares porque este no cambió en el grupo evaluado, lo cual no indica que una menor densidad de perfusión no altere las células ganglionares, sino que la reducción de la densidad de perfusión encontrada no afectó el grosor de esa capa neural. Los pacientes evaluados podrían corresponder a un grupo en el cual la alteración vascular precede al daño neural, como plantearon Simó, et al. ${ }^{11}$ y Marques, et al..$^{12}$ en sus estudios.

No encontramos un aumento significativo de los parámetros de la zona avascular foveal en los pacientes con diabetes, lo que ha sido un hallazgo común en los estudios que reportan cambios en el grosor de la capa de células ganglionares ${ }^{13,14}$. Esto podría deberse a que la densidad de perfusión central tampoco fue menor. Rosen, et al. ${ }^{15}$ han propuesto que un cambio muy temprano en la diabetes es un aumento en la densidad de capilares perfundidos como mecanismo compensatorio, particularmente los más cercanos a la zona avascular foveal. Si este fuera el caso en los ojos de nuestro estudio, probablemente estarían en una etapa previa a la disminución del grosor de la capa de células ganglionares, pero confirmarlo requeriría otro tipo de diseño.

Una variable que pudiera haber modificado los resultados del estudio es la edad, que fue mayor en los pacientes con diabetes; sin embargo, el efecto que podría haberse presentado es una pérdida de células ganglionares, lo cual hubiera reducido el grosor de esta capa. Trabajar con grupos con rangos de edad y una duración de la diabetes más acotados permitiría determinar si los hallazgos acerca de las correlaciones encontradas en este estudio son consistentes. Otra limitación podría ser la hipertensión arterial, pero en los sujetos que la padecen la característica que se asocia con una duración prolongada de la enfermedad es la disminución del grosor de la capa de células ganglionares ${ }^{16}$.

Una fortaleza del estudio es la medición de la densidad de perfusión por cuadrantes, que permitió identificar las regiones de la parafóvea más afectadas y correlacionar sus valores con los del grosor de la capa de células ganglionares por sector. Una potencial limitación es el tamaño de la muestra, pero el número de pacientes 
evaluados bastó para encontrar diferencias estadísticas en la densidad de perfusión, aunque el grosor de la capa de células ganglionares no cambiara.

La menor densidad de perfusión fue suficiente para mostrar una diferencia estadística; sin embargo, el umbral requerido para encontrar una reducción del grosor de células ganglionares aún se desconoce, y determinarlo requerirá evaluar longitudinalmente pacientes sin retinopatía diabética con mayor duración de la diabetes, porque la asociación entre una reducción de ambas variables en los pacientes con retinopatía diabética ya se ha determinado ${ }^{17-20}$.

\section{Conclusiones}

En los pacientes con diabetes sin retinopatía no se encontró una correlación fuerte entre la densidad de perfusión y el grosor de la capa de células ganglionares, porque este no disminuyó en comparación con los sujetos sin diabetes.

\section{Conflicto de intereses}

Los autores declaran no tener ningún conflicto de intereses.

\section{Responsabilidades éticas}

Protección de personas y animales. Los autores declaran que para esta investigación no se han realizado experimentos en seres humanos ni en animales.

Confidencialidad de los datos. Los autores declaran que han seguido los protocolos de su centro de trabajo sobre la publicación de datos de pacientes.

Derecho a la privacidad y consentimiento informado. Los autores declaran que en este artículo no aparecen datos de pacientes.

\section{Bibliografía}

1. Vujosevic $S$, Aldington SJ, Silva $P$, Hernández $C$, Scanlon $P$, Peto $T$, et al. Screening for diabetic retinopathy: new perspectives and challenges. Lancet Diabetes Endocrinol. 2020;8:337-47.
2. Moshfeghi AA, Lanitis T, Kropat G, Kuznik A, Gibson A, Feng $H$, et al. Social cost of blindness due to AMD and diabetic retinopathy in the United States in 2020. Ophthalmic Surg Lasers Imaging Retina. 2020;51:S6-S14.

3. Tang Z, Chan MY, Leung WY, Wong HY, Ng CM, Chan VTT, et al. Assessment of retinal neurodegeneration with spectral-domain optical coherence tomography: a systematic review and meta-analysis. Eye. 2020 Jun 24. doi: 10.1038/s41433-020-1020-z. Online ahead of print.

4. Furino C, Montrone G, Cicinelli MV, Balestra S, Grassi M, Reibaldi M, et al. Optical coherence tomography angiography in diabetic patients without diabetic retinopathy. Eur J Ophthalmol 2020;30:1418-23.

5. Gildea D. The diagnostic value of optical coherence tomography angiography in diabetic retinopathy: a systematic review. Int Ophthalmol. 2019;39:2413-33.

6. Kim K, Kim ES, Kim DG, Seung-Young Y. Progressive retinal neurodegeneration and microvascular change in diabetic retinopathy: Iongitudinal study using OCT angiography. Acta Diabetol. 2019;56:1275-82

7. Rosenfeld PJ, Durbin MK, Rosiman L, Zheng F, Miller A, Robbins G. ZEISS angioplex spectral domain optical coherence tomography angiography: technical aspects. Dev Ophthalmol. 2016:56:18-29.

8. Lee MW, Lee WH, Ryu CK, Kim TY, Lim HB, Lee YH, et al. Effects of prolonged type 2 diabetes on the inner retinal layer and macular microvasculature: an optical coherence tomography angiography study. J Clin Med. 2020:9:1849.

9. Chansangpetch S, Huang G, Coh P, Oldenburg C, Amoozgar B, He M, et al. Differences in optic nerve head, retinal fiber layer and ganglion cell complex parameters between Caucasian and Chinese subjects. J Glaucoma. 2018;27:350-6.

10. Kim K, Kim ES, Yu SY. Optical coherence tomography angiography analysis of foveal microvascular changes and inner retinal layer thinning in patients with diabetes. Br J Ophthalmol. 2018;102:1226-31.

11. Simó R, Stitt AW, Gardner TW. Neurodegeneration in diabetic retinopathy: does it really matter? Diabetologia. 2018;61:1902-12.

12. Marques IP, Alves D, Santos T, Mendes L, Santos AR, Lobo C, et al. Multimodal imaging of the initial stages of diabetic retinopathy: different disease pathways in different patients. Diabetes. 2019;68:648-53.

13. Li X, Xie J, Zhang L, Cui $Y$, Zhang G, Chen X, et al. Identifying microvascular and neural parameters related to the severity of diabetic retinopathy using optical coherence tomography angiography. Invest Ophthalmol Vis Sci. 2020;61:39.

14. Vujosevic S, Muraca A, Alkabes M, Villani E, Cavarzeran F, Rossetti L, et al. Early microvascular and neural changes in patients with type 1 and type 2 diabetes without clinical signs of diabetic retinopathy. Retina. 2019;39:435-45.

15. Rosen RB, Andrade Romo JS, Krawitz BD, Mo S, Fawzi AA, Linderman $\mathrm{RE}$, et al. Earliest evidence of preclinical diabetic retinopathy revealed using optical coherence tomography angiography perfused capillary density. Am J Ophthalmol. 2019;203:103-15.

16. Santos AR, Ribeiro L, Bandello F, Lattansio R, Egan C, Frydkjaer OU, et al. Functional and structural findings of neurodegeneration in early stages of diabetic retinopathy: cross-sectional analyses of baseline data of the EUROCONDOR project. Diabetes. 2017;66:2503-10.

17. Lupidi M, Coscas G, Coscas F, Fiore T, Spaccini E, Fruttini D, et al. Retinal microvasculature in nonproliferative diabetic retinopathy: automated quantitative optical coherence tomography angiography assessment. Ophthalmic Res. 2017:58:131-41.

18. Park JJ, Chung CS, Fawzi A. Visualizing structure and vascular interactions: macular nonperfusion in three capillary plexuses. Ophthalmic Surg Lasers Imaging Retina. 2018;49:e-182-90.

19. Jung JJ, Yu DJG, Zeng A, Chen MH, Shi Y, Nassisi M, et al. Correlation of quantitative measurements with diabetic disease severity using multiple en face OCT angiography image averaging. Ophthalmol Retina. 2020;4:1069-82

20. Lim HB, Lee MW, Park JH, Kim K, Jo YJ, Kim JY. Changes in ganglion cell-inner plexiform layer thickness and retinal microvasculature in hypertension: an optical coherence tomography angiography study. Am J Ophthalmol. 2019;199:167-76. 